\title{
La frontière des États-Unis et la révolution française : l'exemple de Cincinnati
}

\section{Lee Baker}

\section{(2) OpenEdition \\ 1 Journals}

\section{Édition électronique}

URL : https://journals.openedition.org/ahrf/10062

DOI : 10.4000/ahrf.10062

ISSN : 1952-403X

Éditeur :

Armand Colin, Société des études robespierristes

\section{Édition imprimée}

Date de publication : 15 mars 2006

Pagination : 147-162

ISSN : 0003-4436

\section{Référence électronique}

Lee Baker, «La frontière des États-Unis et la révolution française : l'exemple de Cincinnati », Annales historiques de la Révolution française [En ligne], 343 I janvier-mars 2006, mis en ligne le 01 mars 2009, consulté le 23 avril 2022. URL : http://journals.openedition.org/ahrf/10062 ; DOI : https://doi.org/ 10.4000/ahrf.10062

Ce document a été généré automatiquement le 23 avril 2022.

Tous droits réservés 


\title{
La frontière des États-Unis et la révolution française : l'exemple de Cincinnati
}

\author{
Lee Baker
}

1 Selon une idée répandue et partagée par beaucoup d'Américains, la vie à la frontière à la fin du dix-huitième siècle était marquée par l'isolement, et une solitude aussi importante que l'immensité des espaces du Territoire du Nord-Ouest. Certes, la monotonie de ce quotidien en marge de la civilisation pouvait être brisée par les nouvelles d'un journal. Ainsi, le monde extérieur, lequel devait sembler bien loin pour la plupart des colons, ne l'était jamais vraiment, car la presse permettait de maintenir le contact avec le littoral oriental. Aussi, les journaux relevaient davantage du simple divertissement : ils étaient (et sont) les sources de la nouvelle, un support essentiel dans la formation d'une opinion publique ${ }^{1}$. Avant même leur diffusion, les nouvelles étaient commentées par des amis ou par des connaissances dans des cercles restreints proches des comités de rédaction. Ce partage de l'information, cette manière de faire et de voir a certainement contribué à renforcer les liens au sein des communautés locales. Dans ce processus, la presse participait à la création d'un consensus sur la façon d'aborder et de traiter des problèmes communs ${ }^{2}$. En définitive, les journaux peuvent être considérés comme des sources de première main pour étudier comment les communautés ont appréhendé les difficultés auxquelles elles ont été confrontées.

Cet article examine la conscience collective des habitants de Cincinnati à travers le prisme d'un journal, l'unique journal du Territoire du Nord-Ouest au début des années 1790. Il s'agit, en particulier, de s'attacher à décrire les relations avec les Indiens d'Amérique dans une époque marquée par l'avènement de la Révolution française. La problématique retenue s'articule autour de l'étude des articles de presse traitant de la Révolution française et de leur incidence sur les mentalités à Cincinnati, notamment en ce qui concerne l'ébauche de comparaisons avec la gestion par les Français des populations indigènes ou la pertinence des nouvelles politiques censées améliorer la qualité de la vie dans la petite ville américaine. 
Cincinnati est l'une des villes les plus vieilles du Midwest. Fondé en 1789 (sous l'appellation de Losantiville), ce bourg est rapidement devenu un centre de commerce et la capitale du Territoire du Nord-Ouest. Avant 1793, Cincinnati avait environ 900 habitants, et près de 200 soldats stationnés au Fort Washington. Il y avait une église presbytérienne, mais aucune construction de brique: la ville toute entière a été façonnée à l'aide de rondins. En fait, il n'y avait aucun music-hall, théâtre ou autre centre culturel. Mais il y avait un journal, le Centinel of the North Western Territory, qui imprima sa première édition le 9 novembre 1793 dans un petit chalet entre Sycamore et Front Street : sa présence mérite d'être soulignée pour une ville de la taille de Cincinnati. Certes, dans les années 1790 , les États-Unis avaient le nombre de quotidiens le plus élevé dans le monde: un pour cinquante personnes. Chaque communauté, indépendamment de sa taille, avait son journal propre sur le modèle du The Gazette of the United States (Philadelphie) ou The National Gazette (New York) ${ }^{3}$. Pourtant, les premières années de Cincinnati n'étaient pas des plus propices. Il a été nécessaire pour ses habitants de s'assurer la maîtrise d'espaces gigantesques, forêts hostiles et plaines fertiles. Comme la plupart des villes à la frontière, Cincinnati entretenait un rapport étroit avec les Indiens d'Amérique, et ce n'était pas un hasard si la première construction importante fut le Fort Washington, édifié en 1789. Cette place-forte dominait toujours physiquement la ville dans les années 1790 et elle jouait un rôle dissuasif notable dans l'espace régional. Comme la France en 1793, Cincinnati était en guerre: le Territoire était officiellement partie intégrante des États-Unis d'Amérique, mais l'armée était régulièrement confrontée aux raids des Indiens qui peuplaient le secteur. En 1791, un contingent basé à Cincinnati, commandé par Arthur St. Clair, subissait une grave défaite face aux troupes indiennes de Little Turtle.

4 Lors de cette fameuse confrontation, 900 soldats de l'Ohio occidental étaient portés disparus tandis que les survivants s'étaient enfuis en ordre dispersé, loin du camp retranché de Cincinnati. Les années suivantes, les Indiens, forts de leur succès, poursuivaient les colons jusqu'à la rivière de l'ohio qu'ils considéraient comme la véritable frontière avec les États-Unis. Naturellement, le Centinel ne pouvait éluder la question indienne. Néanmoins, un autre événement, loin de la frontière américaine, intéressait ses rédacteurs : la Révolution française. L'établissement d'une corrélation entre deux phénomènes de part et d'autre du Grand Océan pouvait sembler étrange, mais il ne faudrait pas oublier que les guerres indiennes ont connu leur apogée sur la frontière occidentale à l'époque où les révolutionnaires français prenaient l'ascendant sur leurs ennemis. En outre, la présence française dans la vallée de l'Ohio était demeurée importante car les Français avaient été parmi les premiers européens à explorer cette région reculée. En définitive, rapprocher les guerres indiennes et l'évolution de la Révolution en France n'était pas un exercice incongru pour les rédacteurs de Cincinnati: cette habitude affichée dans les colonnes du Centinel, expression d'une intention délibérée ou au contraire d'une attitude irréfléchie ${ }^{4}$, mérite certainement d'être étudiée.

5 Le fondateur du Centinel of the North Western Territory était William Maxwell. Il était originaire de Lexington (Kentucky) où il avait contribué avec John Bradford à la publication de la Kentucky Gazette. Thomas Léonard a déjà relevé que le journalisme au dix-huitième siècle était « une affaire d'arrivistes » et indiqué qu'un rédacteur du New Jersey s'était plaint en 1802 qu'» un journal régional soit souvent poussé jusqu'à l'épuisement pour assurer sa subsistance $»^{5}$. 
6 Maxwell connaissait les difficultés liées à la fabrication d'un journal dans une petite communauté et avouait se contenter «de petits bénéfices $»^{6}$. Il semble avoir réussi suffisamment pour reconstruire l'église presbytérienne en 1794 à l'aide d'un fonds populaire dont la contribution moyenne était de 2,70 \$7. Toutefois, Maxwell se lassa rapidement du journalisme et vendit cette publication en 1796 à Edmond Freeman, laquelle devint aussitôt le Freeman's Journal. L'histoire de William Maxwell, décédé en $1809^{8}$, et celle du Centinel de Cincinnati étaient étroitement associées depuis ce 9 novembre 1793 où la première édition fut publiée avec une proposition d'abonnement de 2,50 \$ par an (au lieu de sept cents pour l'édition hebdomadaire). L'objet explicite de ce journal prétendument «de grande utilité à la ville » était de fournir l'information sur « ce qui se passe à l'est de l'Atlantique [autrement dit, en Europe] dans les armes et dans les arts de paix " aussi bien que " les transactions différentes au sein des États de l'Union ». Ainsi, le journal s'identifie lui-même comme un vecteur de la connaissance, une attitude courante lors de cette période agitée de fin de siècle. Le Centinel ne cachait pas les difficultés et les périls qui menaçaient Cincinnati, mais considérait comme une priorité le fait d'aborder les questions politiques, sociales et économiques : placée au service de la connaissance et du bien-être commun, l'information devait être accessible pour profiter au plus grand nombre. Dans l'actualité du journal, la question indienne demeurait assurément un problème épineux ${ }^{9}:$ "Les habitants sont quotidiennement exposés à un ennemi qui [...] a balayé des familles entières ». Avec le recul, Maxwell envisageait d'utiliser la perspective européenne comme une source d'inspiration dans la gestion des relations avec les Indiens d'Amérique. Désireux de traiter les problèmes de l'aire régionale d'une manière presque scientifique, il utilisait son journal à cet escient dans la grande tradition des esprits éclairés des Lumières. Il n'y avait alors ni agence de presse, ni journalistes dignes de ce nom ou services télégraphiques.

7 La plupart du temps, les rédacteurs décrivaient des histoires qui ont marqué l'actualité locale et ses environs, en utilisant les témoignages, écrits ou verbaux. D'autres rédacteurs utilisaient des méthodes qui seraient apparentées au plagiat de nos jours puisqu'ils se contentaient de reprendre des articles déjà imprimés dans d'autres journaux. Les nouvelles internationales étaient bien souvent puisées dans des courriers et dans ce cas, le rédacteur était seul juge pour autoriser leur publication. Si les preuves manquent, il est avéré que Maxwell a autorisé par sa signature la parution d'articles déjà publiés à New York, Philadelphie, Lexington, Londres ou Paris : la souscription de journaux britanniques ou français depuis l'intérieur du pays apparaît en effet improbable en raison du coût prohibitif de cette opération. En revanche, la reprise des articles du Kentucky Gazette, le seul autre journal à l'ouest de l'Alleghenies, apparaît avérée. Parmi ceux-ci, les nouvelles traitant des affaires indiennes retiendront ici toute notre attention.

8 En 1793, Cincinnati était à peine plus qu'un ensemble de chalets en bois au sud-ouest de Fort Washington. Le fortin, composé de rondins carrés et de maisons à deux niveaux avait été érigé pour servir de base aux opérations militaires contre les tribus hostiles du Territoire du Nord-Ouest ${ }^{10}$. La ville avait été créée à l'occasion d'une distribution de lots en janvier 1789 : les propriétaires terriens avaient découpé leurs possessions en parts et avaient distribué, en suivant les règles d'une loterie, plusieurs lots aux participants. Les in-lots, au sud de Seventh Street, étaient de la même taille que les out-lots, mais ils avaient été divisés en huit parts d'une moindre importance. Les out-lots étaient utilisés seulement comme terres agricoles en raison de la crainte de raids indiens et, de fait, 
étaient peu développés jusqu'en $1802^{11}$. Il était encore risqué de voyager en direction du nord en raison des attaques fréquentes contre les stations et les convois... et cette restriction de la liberté de mouvement était un problème économique sérieux car le commerce était alors, selon la formule même d'un contemporain, « l'élément vital de la ville $»^{12}$.

La description de Cincinnati comme entrepôt commercial était fréquente. À l'été 1792, Johann Heckewelder, missionnaire auprès des tribus indiennes de la région, visitait Cincinnati qu'il percevait comme une ville «envahie de marchands et de marchandises », une ville qui "s'est remplie pour l'essentiel de mauvaises personnes ». De même, François Baily en 1796 décrivait une grande ville qui servait à la frontière comme "un grand dépôt " pour les marchandises, qu'il comparait à un désert... exception faite des habitations le long du Front, Sycamore et Main Street (parties de la ville situées de nos jours sous un parking) ${ }^{13}$. Daniel Drake a relevé que malgré la modestie de Cincinnati, la plupart des pionniers installés s'y plaisaient : ils adoptaient d'autant plus facilement la vie urbaine lorsqu'ils étaient eux-mêmes originaires, non pas de communautés rurales, mais des villes de l'Est. En fait, ces soi-disant «néocitadins" n'étaient que rarement des fermiers, mais plutôt des marchands ou des négociants déjà marqués par l'urbanité ${ }^{14}$. Ensuite, les établissements de détail et de gros ont contribué à étendre le rayonnement économique de Cincinnati dans les régions avoisinantes. Les difficultés n'étaient pas minces : les espoirs de développer les liaisons commerciales entre la frontière et la côte Est ont été maintes fois brisés, quand un convoi était pillé ou lorsque les employés d'une compagnie commerciale étaient blessés ou laissés pour mort. Dès lors, la sécurité et le renforcement de la loi ont été des préoccupations quotidiennes. Le rôle des institutions et le développement de la civilisation américaine dans le Grand Ouest ont été soulignés par Richard Wade, lequel insistait en particulier sur les transferts culturels et les rapprochements économiques opérés entre l'intérieur sauvage du pays et sa frange littorale déjà modernisée. L'un des premiers signes de ce développement était la création d'un journal populaire ${ }^{15}$, et en la matière, Cincinnati ne dérogeait pas à la règle. Ce journal, le Centinel, n'était ni une création artificielle ni un bien culturel importé, mais un organe de communication sur la frontière, le reflet d'une société américaine en plein essor dans le Territoire du NordOuest.

Dans le tout premier numéro, Maxwell avait indiqué sa volonté de promouvoir des solutions raisonnables pour régler les problèmes de Cincinnati. Mais les recettes miracles, le journal ne pouvait les inventer en dépit des attentes des citoyens. Surtout, la tentation était grande de comparer Cincinnati aux autres centres urbains de l'Ouest car les difficultés étaient analogues, même si cette démarche semblait plus démoralisatrice qu'efficace car les différents problèmes nécessitaient presque toujours des réponses spécifiques.

11 La Révolution française était aussi une source d'inspiration importante du Centinel, avant tout parce qu'elle était une expérience permanente depuis 1789 sur la manière d'administrer un État ou la façon d'affronter les dangers à l'intérieur comme à l'extérieur du pays. À l'extérieur, entre 1792 et la dernière publication du Centinel en 1796, la France était en guerre avec l'Angleterre, la Prusse, l'Autriche et les principautés germaniques. Les armées des plus grandes monarchies d'Europe, des troupes régulières commandées par des généraux aguerris, convergeaient vers les frontières du pays. À l'intérieur, l'éruption de la guerre civile en France en 1793 était le 
résultat d'une crise sans précédent, de l'apparition de périls imminents aux frontières, de bouleversements politiques, administratifs et économiques majeurs; en outre, les régions de l'Ouest (la Vendée) et du Sud-Est menaçaient durant tout l'été la poursuite même de la Révolution. L'acharnement des révolutionnaires français sous l'autorité de Robespierre à continuer le combat contre les régimes monarchiques était un modèle pour Maxwell : peu importent les moyens les plus sanglants, la guillotine et la Terreur, seule comptait l'héroïque défense de la patrie contre les ennemis, tous les ennemis. Ainsi, le Centinel n'hésitait pas à juxtaposer dans un même numéro la description des diverses opérations militaires en France et les combats contre les Indiens dans le Territoire du Nord-Ouest. À cette époque, en 1793, le général Anthony Wayne préparait les troupes du Fort Washington pour une expédition dans le Nord.

Après la débâcle en 1791, St. Clair, demeuré gouverneur, avait néanmoins été remplacé au poste de commandant militaire par le général Wayne qui entraînait ses troupes pour une grande campagne d'épuration des belliqueux Peaux-Rouges de la vallée de l'Ohio. Maxwell, tel un reporter de guerre, rapportait les grandes étapes de cette campagne : Wayne, partant de Cincinnati à l'automne 1793 à la tête de 3000 soldats, les premières escarmouches sérieuses l'année suivante et la progression vers le Nord. Lorsque cette guerre indienne atteignit son apogée, Maxwell relata comment les Français avaient adopté des mesures radicales, voire désespérées pour contrer les assauts de leurs adversaires. Par exemple, le 21 décembre 1793, le journal narrait comment la France, pour mobiliser ses ressources, avait décrété le 28 août « l'état de siège »; ce même jour, il était précisé qu'un colon avait été tué par les Indiens et que deux autres patriotes étaient blessés à la suite d'une attaque dans les faubourgs de la ville. Une semaine plus tard, on apprenait que les soldats édifiaient un poste dans ce secteur pour s'assurer le contrôle "[...] d'un grand village de Miami [qui avait] été trouvé »" ${ }^{16}$. Deux semaines après cet incident, il était annoncé que Bertrand Barère, un membre du Comité de Salut public avait répondu le 23 août au soulèvement de l'ouest de la France, en Vendée, en préconisant la levée en masse tandis que la ville rebelle de Toulon rejoignait l'insurrection et que ses insoumis déclaraient vouloir abattre chaque émissaire de Paris. Dans cette même édition, Maxwell misait une nouvelle fois sur la juxtaposition des événements en donnant de l'écho à une attaque du Fort Hamilton (deux colons disparus et un présumé mort) ${ }^{17}$. Cet hiver là a été marqué par certains changements adoptés par les Français dans leur lutte contre les contre-révolutionnaires. Aussi, le Centinel évoquait le gouvernement révolutionnaire français qui intentait un procès à Marie-Antoinette afin de freiner les ardeurs des royalistes.

13 La reine était accusée de fomenter la rébellion, de comploter contre la nouvelle République et de communiquer avec l'ennemi en temps de guerre. Reconnue coupable, le Centinel annonçait l'exécution du 16 octobre: "Hier matin Marie-Antoinette puissante et belle, l'épouse de Louis malheureux, le roi de France, a été apportée comme le malfaiteur le plus commun de la prison de la Conciergerie et placée à la barre $\mathrm{du}$ tribunal révolutionnaire. Ce matin, cette victime malheureuse de la fureur démocratique a été honteusement portée à la place d'exécution dans un chariot. La force armée de Paris était à pied du Palais de Justice jusqu'à la Place de la Révolution. Alors qu'elle passait, la multitude s'est écriée "bravo" ». Les mesures radicales adoptées par les Français étaient véhiculées dans les colonnes du quotidien au fur et à mesure que les nouvelles de la guerre indienne parvenaient à Cincinnati. Aussi, dans ce même numéro, l'attaque d'un chariot à neuf miles au sud du Fort Hamilton (vingt miles au nord de Cincinnati) était abondamment commentée ${ }^{18}$. Le 5 avril 1794, un correspondant 
du Centinel notait que Colombia, un poste d'observation non loin de là, offrait des primes pour des scalps d'indiens. Il y avait une autre attaque juste deux semaines plus tard: «Beaucoup de rapports ont circulé en ce qui concerne les attaques faites par les sauvages sur un convoi de fournitures, il y a quelque temps, entre le fort st. Clair et fort Jefferson. Voici un compte-rendu authentique de cette affaire. Les Indiens ont emporté environ soixante-dix chevaux et ont laissé les chariots debout sur la route. Cette nuit, un parti d'environ quarante ou cinquante Indiens a fait une attaque sur la station de White et a tué un des hommes et deux des enfants tandis que les autres se sont enfuis $»^{19}$. Cette affaire ne s'ébruita plus jusqu'au 3 mai, jour de plusieurs attaques simultanées sur trois convois qui firent deux morts et plusieurs blessés graves ${ }^{20}$. Dès lors, les demandes pour la prime au scalp ne cessèrent d'augmenter, surtout après une nouvelle attaque deux semaines plus tard contre une colonne de soldats (huit tués), des troupes pourtant bien formées et armées. En conséquence, la ville adoptait la pratique du scalp (oreille droite comprise pour éviter la division par moitié) et son journal détaillait dans un encart les primes versées (entre 100 et 136 dollars) dans cinq numéros consécutifs ${ }^{21}$. Les Français n'avaient évidemment pas ce genre de problèmes, mais l'efficacité de leurs politiques pour la défense de la Patrie avait été largement médiatisée par le Centinel: la politique dite de la "Terreur", même si elle n'était mentionnée expressément, était perçue dans le journal comme une mesure aussi novatrice que salutaire pour la France. Pourtant, seuls ses principaux traits étaient connus, et encore, par l'intermédiaire de bribes issues de journaux français et britanniques reproduites à New York ou Philadelphie. Dans ces «histoires", l'information factuelle n'était pas privilégiée, mais plutôt la manière dont les Français surmontaient leurs difficultés dans une guerre brutale et impitoyable, une guerre totale sur tous les fronts.

Outre la description d'un contexte général défavorable et la trame des initiatives françaises, le journal s'attardait sur les mesures spécifiques adoptées dans le pays des Droits de l'Homme contre les rebelles à Toulon, Lyon ou en Vendée. Dans l'édition du 18 janvier 1794, Maxwell choisissait les articles sur la rébellion à Lyon où « la moitié de la ville est en flammes » sur la bataille de Toulon déjà en voie d'achèvement, et l'ouest "purgée de tous les aristocrates... Bientôt il n'y aura plus de Vendée $»^{22}$. Quelques semaines plus tard, le Centinel annonçait que la Convention nationale avait ordonné « la destruction complète de Lyon» et que ses ruines seraient rebaptisées "Ville Affranchie. $»^{23} \mathrm{Il}$ annonce que "les citoyens, les troupes de la république sont triomphalement entrés dans Lyon, le 19e jour de ce mois. Vous avez invité votre comité d'assistance publique à prendre de nouvelles mesures sur ce compte. Elles ont été prises et j'ai été chargé de vous les expliquer... Les chefs des rebelles, au nombre de 2 000, ont tenté de se sauver, mais ils sont poursuivis sur tous les côtés et ne peuvent pas échapper non seulement à l'ardeur de nos troupes, mais aussi à celui des gens de Lyon. J'ai retenu le courrier pour vous informer que les rebelles ont été poursuivis et mis en pièces sur tous les côtés. ${ }^{24} \mathrm{Il}$ doit être noté qu'il n'était pas fait mention des motivations des contre-révolutionnaires, car seul l'aspect de la répression semblait passionner Maxwell. Dès lors, la politique était réduite à sa plus simple expression, comme ce 15 mars 1794, lorsqu'il était relevé que «Cent trente députés ont été arrêtés, plusieurs ont été guillotinés et d'autres sont en procès $»^{25}$. En définitive, le Centinel ne parvenait pas à expliquer les fondements de la Révolution française, ses valeurs, les réactions qu'elle suscitait... et certaines notions ou termes politiques (Montagne, Girondins, Plaine) auraient mérité d'être explicités. Il est vrai que le leitmotiv de la 
publication n'était pas la cause révolutionnaire, mais la soumission des rebelles en France. Le seul article sur l'arme politique de la Terreur était la reproduction d'un discours de Robespierre sur sa légitimité : sa publication en première page le 12 avril 1794 ne devait rien au hasard car celui-ci évoquait comment la patrie devrait traiter ses plus farouches ennemis ${ }^{26}$; en substance, ce discours insistait sur le fait qu'aucune pitié ne devait être accordée et que toute énergie devait être utilisée au service de la cause de la nation. Ainsi, les facteurs politiques ou religieux de la Révolution ont été minorés au détriment de l'événementiel... parfois jusqu'à l'anecdotique, comme lorsque le Centinel annonçait que le gouvernement français avait découvert une grande cachette d'objets d'or et précieux à la cathédrale de Saint-Denis et que son contenu avait été confisqué pour financer l'effort de guerre ${ }^{27}$.

15 Entre la Révolution française et les États-Unis, entre l'ancien monde et le nouveau, il y avait un lien apparent, la politique de l'ambassade de France: en 1793, Edmond Genet avait été envoyé par le gouvernement révolutionnaire pour représenter les intérêts français aux États-Unis. Des intérêts politiques et commerciaux liaient les deux Républiques, tandis que sur le plan militaire, il fallait coordonner les efforts contre les Anglais et les Espagnols dans le but de favoriser la libération de l'Amérique Latine ou d'ouvrir le Mississippi aux explorateurs du Kentucky. Un vent de liberté soufflait dans les pages du Centinel lorsqu'il s'agissait d'évoquer la situation en France, dans le Kentucky, la Floride ou la Louisiane ${ }^{28}$. Genet, dans l'accomplissement de sa mission, s'est engagé dans une variété d'activités le long de la façade orientale pendant le printemps de 1793, période où l'amitié franco-américaine s'était détériorée ${ }^{29}$. Un des agents de Genet, André Michaux, avait essayé de lever une armée sous la direction de George R. Clark pour des opérations dans l'Ouest contre les Anglais en Louisiane. Il appelait les volontaires à le rejoindre dans un encart du Centinel le 25 janvier 1794 en promettant 1000 acres par expédition, 2000 acres pour un service d'une année et 3000 pour deux années d'engagement, ou $1 \$$ par jour pour les moins prévoyants ${ }^{30}$. Le brasdroit de Genet encourageait donc les milices américaines à attaquer des postes anglais provoquant une situation inconfortable pour la diplomatie américaine et la politique de la neutralité. Sans surprise, peu de volontaires se lancèrent dans l'aventure hasardeuse de Michaux. Si le Centinel a été fondé trop tard pour soutenir toutes les activités de Genet, il s'était néanmoins engagé à soutenir quelques-unes de ses initiatives, quitte à défendre Genet en proie aux critiques de gens importants. L'effort était modeste, le journal ne publiant que les articles où Genet apparaissait irréprochable, comme cette lettre du 28 décembre 1793 adressée au Procureur Général des États-Unis, Edmond Randolph, dans laquelle il se plaignait d'être diffamé et indiquait sa volonté de poursuivre en justice ses détracteurs, messieurs Rufus King et John Jay ${ }^{31}$.

16 En parallèle, Maxwell soutenait les efforts de Michaux pour lever une armée. Conscient de cette situation, St. Clair, gouverneur du Territoire du Nord-Ouest, plaçait lui aussi une publicité dans le Centinel: il mettait en garde tous les Américains contre les agissements des agents français (Laclaire, Delpeau, Mathieu, Signeux) en rappelant à quel point il était grave de menacer la neutralité des États-Unis. L'influence de St Clair était significative puisque sa déclaration fut imprimée en première page six semaines consécutives $^{32}$. En décembre 1793, le Président Washington, lassé de l'intervention de Genet dans des affaires américaines, annonçait son intention de se passer de ses services; la chose fut acquise le 21 février $1794^{33}$. Cette nouvelle n'était pas arrivée à Cincinnati avant le 31 mai 1794, quand le Centinel imprimait un communiqué de J. Fauchet, le remplaçant de Genet, indiquant que «toutes les missions violant la 
neutralité des États-Unis sont annulées. $~^{34}$ Si l'affaire Genet n'offre pas d'exemple sur la façon de combattre les Indiens, elle dévoilait l'attitude des Français prêts à utiliser toute méthode pour défaire leurs ennemis. Certes, la partialité de Maxwell ne pouvait être masquée puisque rien n'a jamais été publié de critique sur Genet, tandis que ses déclarations d'innocence et ses bonnes intentions trouvaient une place de choix dans son journal. Les lecteurs demeuraient attentifs aux affaires internationales et aux affaires françaises : le Kentucky et l'Ohio disposaient d'une longue tradition francophile et ceci, les rédacteurs du Centinel ne pouvaient l'ignorer. Par exemple, le 1er mars 1794, le journal imprimait une table de correspondance entre les calendriers français et américain, laquelle, jamais utilisée dans Cincinnati, témoignait d'un intérêt pour les affaires françaises. S'agissant des subtilités propres aux affaires politiques françaises, elles étaient probablement trop complexes pour le lecteur américain et étaient de fait ignorées dans le Centinel. Une comparaison avec d'autres journaux américains démontre la volonté de Maxwell de se concentrer sur la Révolution et ses aspects militaires parce que ceux-ci étaient utiles dans la lutte contre les Indiens.

Observons la couverture médiatique de ces événements dans deux autres journaux, Kentucky Gazette (de Lexington, une autre ville de la frontière) et Newport Mercury (Newport, Rhode Island, à l'opposé de Cincinnati) ${ }^{35}$. Maxwell avait déjà travaillé à la Kentucky Gazette en utilisant les mêmes réseaux, les mêmes sources qu'à Cincinnati : très tôt, il s'était spécialisé dans la nouvelle de guerre dans un journal où les problèmes de la frontière émergeaient de manière comparable. Le Mercury couvrait également la Révolution, mais le traitement des événements se différenciait nettement des journaux du Kentucky et de l'Ohio. Les aspects politiques étaient différemment privilégiés depuis l'ouverture des états généraux en mai 1789: en comparaison, la couverture des prémisses de la Révolution par le Kentucky Gazette était plutôt superficielle ${ }^{36}$ tandis que seul le Mercury explicitait la chute de Robespierre en $1794^{37}$. La Terreur était en définitive un phénomène français dont la complexité échappait aux lecteurs du Centinel et, à un degré moindre, à ceux du Mercury car celui-ci prenait la peine d'évoquer ses tenants et aboutissants ${ }^{38}$.

De l'ennemi et de la façon de le combattre, il était toujours question à l'hiver 1794 tant en Amérique que sur le vieux continent. Sur la frontière américaine, le temps des victoires était amorcé tandis que la menace indienne reculait, tout comme le péril pour la France des armées coalisées. Le 1er février 1795, Maxwell n'hésitait pas à décrire « la France, sans tous les envahisseurs ${ }^{39}$. Il encourageait la publication d'une lettre du Comité de Salut public adressée au Congrès américain (datée du 26 avril 1794). Le Comité, saluant les victoires sur les champs de bataille, louait les mérites de l'amitié entre les deux républiques : "nos succès réverbèrent sur vous " et " notre cause est réciproque ». "L'aristocratie est mise en fuite, écrit la société populaire de Belfort. Le royalisme est annihilé. Les mécontents et les traîtres sont partout poursuivis. La superstition est dans le désespoir, le fanatisme a disparu, la raison gouverne ; l'assignat est à la parité, l'impôt va; les autorités constitutionnelles sont jusqu'à leurs grands principes, les gens sont calmes; les sociétés populaires sont reconstituées... Si vous demandez, des législateurs, qui ont fait fonctionner ces prodiges : c'est Herault... Le prince de Hohenlohe avait appelé le commandant de Landau pour livrer la place ; mais Laubadere et ses frères courageux dans des armes ont répondu au prince dans le style de vrais républicains ». Tout porte à croire que, si l'occasion s'était présentée à lui, 
Maxwell aurait répondu à cette lettre en annonçant aux Français le succès de la campagne contre les Indiens.

À l'été 1794, le général Wayne avait déjà réduit le potentiel offensif des tribus indiennes lors de la bataille de Fallen Timbers où les Indiens avaient été contraints d'accepter les clauses du traité de Greenville (abandon de presque tout l'Ohio et d'une partie de l'Indiana). Par ce traité, reproduit à maintes reprises en première page du Centinel, le Territoire du Nord-Ouest s'assurait une certaine sécurité... sécurité à laquelle St. Clair tenait énormément, n'hésitant pas à menacer quiconque s'en prendrait dorénavant aux communautés indiennes ${ }^{40}$. Les nouvelles lois étaient imprimées dans le journal de Cincinnati, nécessitant même l'ajout de nouveaux suppléments ${ }^{41}$. Valoriser les ressemblances entre la France et la frontière américaine était plus que jamais la volonté d'un Maxwell toujours sensible aux événements révolutionnaires, comme la nouvelle constitution française de 1795 , d'autant plus que cette dernière avait été rendue possible par les victoires militaires des années précédentes. Chroniqueur, journaliste et éditeur, Maxwell a réimprimé la constitution toute entière en première page de nombreuses fois à côté des extraits des lois du Territoire du Nord-Ouest : il ne pouvait s'agir d'une coïncidence ${ }^{42}$. En France comme dans le Territoire du Nord-Ouest, l'heure était à la réorganisation administrative, un thème secondaire dans le Centinel. $\mathrm{Au}$ fil des ans, le journal prenait une importance croissante sur la frontière de l'Ohio en soutenant les colons de l'Ouest sauvage, en publiant les nouvelles de Cincinnati, en recherchant de l'inspiration dans l'histoire de la Révolution française. Ainsi, grâce au Centinel, la vallée de l'Ohio se fascinait dans les années 1790 pour cette «autre Révolution » que l'Amérique avait connue une dizaine d'années auparavant. Toutefois, de cette Révolution française, le journal de Cincinnati avait surtout retenu l'aspect strictement militaire et la défense héroïque de ses partisans face à d'impitoyables ennemis. S'efforçant de décrire cette lutte désespérée pour la survie, le Centinel pourrait être comparé à un miroir à deux faces : l'une dédiée aux succès français, l'autre dédiée aux succès américains.

\section{BIBLIOGRAPHIE}

Sources PrimairesNon publiées :

Cincinnati Historical Society Library, Cincinnati, OH. Centinel of the North Western Territory, 071.771, fc5ce, R.B.

Newberry Library, Chicago, IL. The Kentucky Gazette, A6.49.

Newberry Library, Chicago, IL. Newport Mercury, +A6.625.

Publiées :

Bailly, Francis. Journal of a Tour in Unsettled Parts of North America, 1796 \& 1797. Edited by Jack Holmes. Carbondale, Southern Illinois University Press, 1969.

Drake, Daniel. Pioneer Life in Kentucky, 1785-1800. Edited by Ernest Field Hoine. New York, Henry Schuman, 1948.

Annales historiques de la Révolution française, 343 | janvier-mars 2006 
Heckewelder, J. The First Description of Cincinnati and other Ohio Settlements : The Travel Report of Johann Heckewelder (1792). Edited by Don H. Tolzmann. Lanham, University Press of America, 1988. Record of the Distribution and Sale of Lots in the Town of Losantiville (Now Cincinnati), 1789. Cincinnati, R.A. Cline, 1957.

Rodney, Thomas. A Journey through the West : Thomas Rodney's 1803 Journal from Delaware to the Mississippi Territory. Edited by Dwight Smith and Ray Swik. Athens, Ohio University Press, 1997. Sources Secondaires

Ammon, Harry, The Genet Mission, New York, W.W. Norton \& Company, 1973.

Barker, Hannah and Simon Burrows, Press, Politics and the Public Sphere in Europe and North America, 1760-1820, New York, Cambridge University Press, 2002.

Debo, Angie, A History of the Indians of the United States, Norman, University of Oklahoma Press, 1970.

Dupré, Huntley, « The Kentucky Gazette Reports the French Revolution », The Mississippi Historical Review (XXVI, June 1939-March 1940), p. 163-180.

Greve, Charles, Centennial History of Cincinnati and Representative Citizens, Chicago, Biographical Publishing Company, 1904. 2 volumes.

Hooper, Osman C, The History of Ohio Journalism, Columbus, The Spahr \& Glenn Company, 1933.

Hyslop, Beatrice, « American Press Reports of the French Revolution, 1789-1794 », The New York Historical Society (XLII: 4, October 1958), p. 329-348.

---------, « The American Press and the French Revolution of 1789 », Proceedings of the American Philosophical Society (104:1, February 15, 1960), p. 54-85.

Jackle, John A, Images of the Ohio Valley: A Historical Geography of Travel, 1740-1860, New York, Oxford University Press, 1977.

John, Richard R., Spreading the News: The American Postal System from Franklin to Morse, Cambridge, Harvard University Press, 1995.

Leonard, Thomas C., The Power of the Press: The Birth of American Political Reporting, New York, Oxford University Press, 1986.

Wade, Richard C., «Urban Life in Western America, 1790-1830 », The American Historical Review (64: 1, October 1958), p. 14-30.

-------, The Urban Frontier : The Rise of Western Cities, 1790-1930, Urbana, University of Illinois Press, 1996.

\section{NOTES}

1.Pour une élucidation de ce point, voir Richard R. JOHN, Spreading the News: The American Postal System from Franklin to Morse, Cambridge, Harvard University Press, 1995, p. 37-42.

2.Hannah BARKER et Simon BURRows, Press, Politics and the Public Sphere in Europe and North America, 1760-1820, New York, Cambridge University Press, 2002, p. 4.

3.Beatrice HYSLOP, « American Press Reports of the French Revolution, 1789-1794 ». The New York Historical Society, XLII, 4, October 1958, p. 329. 
4. Voir Angie DEBO, A History of the Indians of the United States, Norman, University of Oklahoma Press, 1970, p. 90-91.

5.Thomas LEONARD, The Power of the Press : The Birth of American Political Reporting, New York, Oxford University Press, 1986, p. 54-55.

6.Voir la première publication Cincinnati Historical Society Library, Cincinnati, Ohio, Centinel of the North Western Territory, 071.771, fc5ce, R.B., page 1 (CHSL).

7.Une table avec les déposants et les sommes est donnée dans Charles GREVE, Centennial History of Cincinnati and Representative Citizens, Chicago, Biographical Publishing Company, 1904, vol. I, p. 361-363.

8.Osman C. HOOPER, The History of Ohio Journalism, Columbus, The Spahr \& Glenn Company, 1933, p. 14-15.

9.CHSL, Centinel, 9 novembre 1793, $\mathrm{n}^{\circ} 1$.

10.John A. JACKLE, Images of the Ohio Valley : A Historical Geography of Travel, 1740-1860, New York, Oxford University Press, 1977, p. 143.

11.GREVE, Centennial History, tome I, p. 336.

12.Johann HECKEWELDER, The First Description of Cincinnati and other Ohio Settlements : The Travel Report of Johann Heckewelder (1792), edited by Don H. Tolzmann, Lanham, University Press of America, 1988, p. 45-46.

13.Francis BAILLY, Journal of a Tour in Unsettled Parts of North America, 1796 \&1797, edited by Jack Holmes, Carbondale, Southern Illinois University Press, 1969, p. 111.

14.Daniel DRAKE, Pioneer Life in Kentucky, 1785-1800, edited by Ernest Field Hoine, New York, Henry Schuman, 1948, p. 15-16.

15.Richard C. WADE, The Urban Frontier : The Rise of Western Cities, 1790-1930, Urbana, University of Illinois Press, 1996, p. 130.

16.CHSL, Centinel, 28 décembre 1793, $\mathrm{n}^{\circ} 8$.

17.Ibid., 4 janvier1794, $\mathrm{n}^{\circ} 9$.

18.Ibid., 8 mars $1794, \mathrm{n}^{\circ} 18$.

19.Ibid., 22 mars 1794, $\mathrm{n}^{\circ} 20$.

20.Ibid., 3 avril 1794, $\mathrm{n}^{\circ} 21$, et 3 mai 1794, $\mathrm{n}^{\circ} 25$.

21.Ibid., 17 mai, $n^{\circ} 27,24$ mai, $n^{\circ} 28,31$ mai, $n^{\circ} 29,7$ juin, $n^{\circ} 30$, et 14 juin, $n^{\circ} 31$.

22.Ibid., 18 janvier $1794, \mathrm{n}^{\circ} 11$.

23.Ibid., 1 mars $1794, \mathrm{n}^{\circ} 17$, et 15 mars $1794, \mathrm{n}^{\circ} 19$

24.Ibid., 22 février $1794, \mathrm{n}^{\circ} 12$.

25.Ibid., 15 mars $1794, \mathrm{n}^{\circ} 19$.

26.Ibid., 12 avril 1794, $\mathrm{n}^{\circ} 22$.

27.Ibid., 10 mai1794, $\mathrm{n}^{\circ} 26$.

28. Harry Ammon, The Genet Mission, New York, W.W. Norton \& Company, 1973, p. 25-29. 29.Le livre d'Amon est une analyse excellente de la mission de Genet.

30.CHSL, Centinel, 25 janvier 1794, n 12 , et AMON, p. 83-85.

31.Ibid., 28 décembre 1793, $\mathrm{n}^{\circ} 8$.

32.28 décembre 1793, $\mathrm{n}^{\circ} 8$ jusqu'au 28 février, $1794, \mathrm{n}^{\circ} 13$.

33.AMON, The Genet Mission, p. 152-153.

34.CHSL, Centinel, 31 mai 1794, $\mathrm{n}^{\circ} 29$.

35.Newberry Library, Chicago, IL (NL), The Kentucky Gazette, A6.49, et Newport Mercury, +A6.625.

36.NL, The Kentucky Gazette, vol. 2-4, p. 236, et Newport Mercury, p. 94, 102, 104. 
37.NL, The Kentucky Gazette, vol. 8, p. 1008-1009, et Newport Mercury, 14 octobre 1794, 22 octobre 1794, et 28 octobre 1794. Voir CHSL, Centinel, 3 novembre 1794, n 52.

38.NL, Newport Mercury, 22 octobre 1793.

39.CHSL, Centinel, 7 juin $1795, \mathrm{n}^{\circ} 30$.

40.Ibid., 5 septembre $1795, \mathrm{n}^{\circ} 94$.

41.Ibid., 10 novembre 1794 jusqu'au 28 mars 1795, nos 53-66, 68, et 72.

42.Ibid., 6 décembre 1794, $\mathrm{n}^{\circ}$ 56, jusqu'au 17 janvier $1795, \mathrm{n}^{\circ} 62$.

\section{RÉSUMÉS}

Le seul journal dans le Territoire du Nord-Ouest pendant les premières années de la Révolution française commence à être imprimé à Cincinnati en 1793. C'était une année centrale pour la Révolution et cet article examine comment ce journal a couvert les événements principaux qui ont eu lieu après sa création. Il suggère que le rédacteur a utilisé la lutte révolutionnaire avec des ennemis internes et externes comme une façon d'encourager des résidants locaux dans leur lutte avec des Indiens d'Amérique hostiles. Les types d'information qu'il a voulu imprimer reflètent surtout des soucis locaux, et non des événements internationaux.

\section{The American Frontier and the French Revolution: The Example of Cincinnati}

The only newspaper in the Northwest Territory during the first few years of the French Revolution begin printing in Cincinnati during 1793. This was a pivotal year for the Revolution, and this article examines how that newspaper covered the major events of the Revolution which occurred after its founding. It suggests that the editor used the Revolutionary struggle with internal and external enemies as a way of encouraging local residents during their struggle with hostile Native Americans. The types of information which he chose to print reflected local concerns, but through international events.

\section{INDEX}

Mots-clés : journaux, Révolution française, États-Unis, Cincinnati, Territoire du Nord-Ouest

\section{AUTEUR}

\section{LEE BAKER}

University of Cincinnati

Raymond Walters College

9555 Plainfield Road

Cincinnati, OH 45236 USA

LeeBakerJr@aol.com 